\title{
Asistencia ventilatoria no invasiva en pediatría
}

\author{
FRANCISCO PRADO A. ${ }^{1}$, PAMELA SALINAS F. ${ }^{2}$, GUSTAVO PIZARRO T. ${ }^{3}$, \\ CAROLINA CAMPOS O. ${ }^{4}$, DANIEL ZENTENO A. ${ }^{5}$
}

1. Neumólogo Pediatra. Hospital Josefina Martínez, Sección Respiratorio, Departamento de Pediatría, Pontificia Universidad Católica de Chile, Programa Asistencia Ventilatoria No Invasiva - MINSAL.

2. Enfermera. Hospital Josefina Martínez, Programa Asistencia Ventilatoria No Invasiva - MINSAL.

3. Pediatra. Hospital de Punta Arenas, Programa Asistencia Ventilatoria No Invasiva - MINSAL.

4. Becada Neumología, Hospital Exequiel González Cortés, Universidad de Chile. Complejo Asistencial Dr. Sotero del Río.

5. Neumólogo Pediatra. Hospital Josefina Martínez, Programa Asistencia Ventilatoria No Invasiva - MINSAL.

\begin{abstract}
\section{Non-invasive ventilatory assistance: Pediatrics considerations}

Non-invasive ventilation (NIV) has extended its use in pediatric setting. Besides acute respiratory conditions, it is also used in chronic diseases associated to ventilatory failure. In the acute setting, PICU constitutes the natural scenery. In chronic conditions, therapy is transferred home as a multidisciplinary program already available in our country. The objective of this review is an update applying respiratory physiology, technical and clinical issues necessary for choosing and using accurately NIV in both acute and chronic settings.
\end{abstract}

(Key words: non - invasive ventilation, acute and chronic settings, technological and clinical issues).

Rev Chil Pediatr 2008; 79 (6): 580-592

\section{RESUMEN}

La asistencia ventilatoria no invasiva (AVNI) es cada vez más utilizada en pacientes pediátricos; tanto en patologías respiratorias agudas, como en enfermedades crónicas que comprometen la bomba respiratoria en forma primaria o secundaria. En el escenario agudo, el paciente requiere de manejo intrahospitalario en Unidades de Paciente Critico (UPC), sin embargo en el paciente crónico su rol se extiende al hogar a través de programas multidisciplinarios integrales disponibles en nuestro país. El presente artículo de actualización pediátrica entrega las bases fisiológicas de la AVNI, sus diferentes modalidades, alternativas de interfases, sistemas de humedificación, aspectos de oxigenación y terapia broncodilatadora. Se entregan aspectos prácticos para la selección y empleo de la AVNI en el escenario agudo y crónico.

(Palabras clave: asistencia ventilatoria no invasiva, niños, domicilio, enfermedades crónicas, insuficiencia respiratoria aguda).

Rev Chil Pediatr 2008; 79 (6): 580-592

Trabajo recibido el 23 de septiembre de 2008, aceptado para publicación el 10 de diciembre de 2008.

Correspondencia a:

Francisco Prado A.

E-mail: panchoprado2004@yahoo.com 


\section{Introducción}

La asistencia ventilatoria no invasiva (AVNI), es una modalidad de ventilación mecánica que se basa en la aplicación cíclica o continua de presión positiva en la vía aérea. No requiere de vía aérea artificial, evitando las complicaciones generadas por un tubo endotraqueal o traqueostomía ${ }^{1}$. Por lo tanto, se utiliza como interfase una mascarilla nasal, naso-bucal o facial y puede ser implementada para el manejo de la insuficiencia respiratoria aguda y crónica ${ }^{1,2}$.

El objetivo es lograr un volumen corriente apropiado dependiendo de la impedancia del sistema, determinada por la resistencia en la vía aérea y distensibilidad de la unidad caja torácica/ pulmón $^{1,2}$. En Unidades de Paciente Crítico Pediátrico (UPC) se utiliza cada vez con mayor frecuencia en el tratamiento de la insuficiencia respiratoria aguda hipoxémica y/o hipercapnica ${ }^{3}$. Se evita la intubación endotraqueal y por lo tanto sus riesgos asociados como son el daño de la vía aérea, barotrauma y neumonía asociada a ventilación mecánica ${ }^{1,3,4}$. En adultos, existen estudios randomizados controlados que apoyan su uso en pacientes con patologías específicas, como reagudización de EPOC y edema pulmonar agudo cardiogénico ${ }^{2,5}$; al ser comparada con la ventilación mecánica invasiva (VMI), demuestra menores índices de mortalidad, días de hospitalización y complicaciones. En niños ha demostrado ser útil en reportes de casos y series de $\operatorname{casos}^{3,6-8}$. Las claves del éxito de la AVNI en pacientes con insuficiencia respiratoria aguda es la cuidadosa selección de los pacientes, monitorización adecuada, disponibilidad del material necesario, experiencia del equipo tratante y contar con protocolos clínicos bien diseñados ${ }^{5,6}$.

La evidencia de los beneficios de la AVNI en las exacerbaciones de pacientes con hipoventilación crónica, en especial portadores de enfermedad neuromuscular y/o cifoescoliosis, es mejor conocida que en pacientes sin comorbilidad $^{9,10}$. Del mismo modo, en estos pacientes, cada vez se suma más información que vincula el inicio de la AVNI precoz, con un mejor pronóstico $^{11-13}$.

En este artículo se abordarán los aspectos fisiológicos y clínicos vinculados a estos dife- rentes escenarios en pacientes con y sin morbilidad de la bomba respiratoria.

\section{Modalidades de AVNI con presión positi- va en la vía aérea}

Existen dos modalidades para entregar soporte de presión positiva con interfases no invasivas ${ }^{13,14}$.

CPAP (continuous positive airway pressure). Es la modalidad de AVNI más sencilla. Se entrega una presión positiva uniforme durante todo el ciclo respiratorio, de tal modo de mantener la vía aérea abierta, aumentar la capacidad funcional residual (CRF) y disminuir el colapso alveolar. La frecuencia respiratoria y el volumen corriente es determinado por el propio enfermo ${ }^{10,14}$.

Es especialmente útil en apnea obstructiva del sueño (SAOS), en patologías con obstrucción de la vía aérea alta y resistencia aumentada secundaria a causas anatómicas (Síndrome de Pierre Robin, Treacher Collins), u obstrucciones dinámicas de la vía aérea (traqueomalacia y laringomalacia $)^{15}$. En patologías restrictivas agudas como edema pulmonar agudo, injuria pulmonar o síndrome de distress respiratorio agudo, la CPAP aumenta la CRF, mejorando las propiedades mecánicas del pulmón al desplazar la ventilación hacia una zona más favorable dentro de la curva presión-volumen, lo que produce reclutamiento alveolar, disminuye el trabajo respiratorio y mejora el intercambio gaseoso $0^{4,5,14}$. En pacientes con patología obstructiva, síndrome de hiperinsuflación pulmonar y auto-PEEP (PEEPi), con colapso dinámico de la vía aérea, el uso de CPAP externo a niveles cercanos al PEEP disminuye el gradiente de presión necesario para generar flujo inspiratorio, disminuyendo el trabajo respiratorio, al ser necesario generar una gradiente de presión pleural menor al inicio de la inspiración.

Para esta modalidad se puede usar cualquier interfase: cánula nasofaringe o nasal, mascarilla naso-bucal o nasal ${ }^{14,16}$. Se recomienda comenzar con valores bajos, de $4 \mathrm{cmH}_{2} \mathrm{O}$, luego ir aumentado de 2 en $2 \mathrm{cmH}_{2} \mathrm{O}$, según tolerancia del paciente y necesidades respiratorias. En general un $\mathrm{CPAP}>\mathrm{a} 12 \mathrm{cmH}_{2} \mathrm{O}$ no es 
bien tolerado y si se requiere es preferible cambiar la modalidad de ventilación ${ }^{16,17}$.

BIPAP (Bilevel positive airway pressure). Entrega presión positiva a la vía aérea en 2 niveles, con ajuste independiente: presión inspiratoria (IPAP) y presión espiratoria (EPAP). La diferencia entre ambas es el nivel de presión de soporte ${ }^{17}$.

IPAP (inspiratory positive airway pressure): Fija el límite de presión inspiratoria. Controla la ventilación, a mayor IPAP se generan mayores volúmenes corrientes durante la fase inspiratoria. El \% del tiempo inspiratorio determinará la duración del IPAP.

EPAP (expiratory positive airway pressure): Fija el límite de presión espiratoria por encima del nivel cero de la presión atmosférica. Mejora la CRF y la oxigenación.

Presión de soporte (IPAP-EPAP): La diferencia entre IPAP y EPAP genera una gradiente de presión. La ventilación se produce como consecuencia de la diferencia entre estas presiones ${ }^{(14,17)}$.

Esta modalidad es útil en trastornos restrictivos que requieren aumentar la CRF y disminuir el trabajo de los músculos respiratorios, como por ejemplo en pacientes con enfermedades neuromusculares (Distrofia muscular de Duchenne, Steiner, Ulrich, Miopatía Congénita, Atrofia Espinal Congénita), cifoescoliosis severa, y en enfermedades con compromiso anatómico del sistema nervioso central como mielomeningocele operado y malformación de Arnold Chiari tipo I y II ${ }^{4,9,11-13,15,18-20}$. Podría emplease también en alteraciones del centro respiratorio (síndromes de hipoventilación central) ${ }^{10,13,15,21}$.

E1 BIPAP se puede utilizar en distintas modalidades, con diferencias entre las ofertas disponibles por los distintos equipos existentes en la actualidad ${ }^{14,21}$.

\section{Modalidades de ventilación no invasiva con BIPAP $10,14,21,22$}

1. Modo S (asistido): Se mantiene un EPAP y cuando el paciente genera una inspiración se gatilla el IPAP programado. La frecuencia respiratoria (FR) y la duración de la inspiración la controla el paciente. El paciente realiza todas las respiraciones y la máquina las apoya.
2. Modo S/T (asistido/controlado): Durante las respiraciones espontáneas el paciente recibe presión de soporte. Si el paciente no realiza un número determinado de respiraciones, o no sensa las respiraciones del paciente, el aparato genera un IPAP y un EPAP a la FR determinada (ciclo automático).

3. Modo T (controlado): El respirador genera los ciclos programados, independiente de las respiraciones espontáneas del paciente, el ventilador proporciona IPAP y EPAP a una FR programada. E1 IPAP es iniciado a intervalos de tiempo predeterminados. En estos intervalos predeterminados el IPAP es entregado por un porcentaje específico del ciclo de tiempo total.

Ej. FR determinada $10 \mathrm{x}$ min., entonces si el ciclo de tiempo total es de 60 s. $60 / 10=6$ s.; y si el $\%$ de IPAP está determinado a $40 \%$ del ciclo total, entonces la IPAP se entregará por 2,4 s.

4.- Modo PC (presión/control): Modalidad en que todos los ciclos gatillados por el paciente son apoyados con la programación de IPAPEPAP, rampa de ascenso (rise-time) y tiempo inspiratorio. A diferencia de la modalidad espontánea, en que el tiempo inspiratorio y relación I:E, es fijada por el centro respiratorio del enfermo, en esta modalidad la duración del ciclo es la programada por el operador.

La presión diferencial (IPAP-EPAP) mayor a $4 \mathrm{cmH}_{2} \mathrm{O}$ mejora la ventilación. Se sugiere iniciar la ventilación con valores de IPAP de 8 a $10 \mathrm{cmH}_{2} \mathrm{O}$ e ir aumentando, según tolerancia, en $2 \mathrm{~cm}$ hasta mejorar el trabajo respiratorio; logrando $\mathrm{SpO} 2$ y $\mathrm{PaCO}_{2}$ adecuados Niveles de IPAP sobre $20 \mathrm{cmH}_{2} \mathrm{O}$ son mal tolerados y son una contraindicación relativa a este método de soporte ventilatorio. El EPAP se inicia con valores de $4 \mathrm{cmH}_{2} \mathrm{O}$, infrecuentemente se sobrepasan los $8 \mathrm{cmH}_{2} \mathrm{O}^{4,10}$. La FR de respaldo es necesaria en todos aquellos pacientes en que existe riesgo o confirmación de apneas o volúmenes minutos bajos ya sea por un comando respiratorio comprometido o por fatiga de la bomba respiratoria. En equipos mas antiguos se puede determinar la FR fijando el tiempo inspiratorio del IPAP, habitualmente es un $33 \%$ del ciclo respiratorio (Relación I:E=1:2). En modelos más nuevos, la FR se fija directamente ${ }^{4,22}$.

El Rise-time, regula la velocidad de entrada 
del aire durante el tiempo inspiratorio, a mayor Rise-time, más demorará en alcanzar el IPAP fijado dentro de un ciclo, mejorando la tolerancia en algunos pacientes. Este tiempo deberá ser corto en aquellos pacientes con disnea secundaria a trastornos ventilatorios restrictivos. Los valores oscilan entre $0,1-0,6 \mathrm{~s}^{14}$. La modalidad $\mathrm{P} / \mathrm{C}$ es útil en pacientes con dificultad en mantener un volumen minuto con volúmenes corrientes (VC) apropiados, ayuda por lo tanto disminuyendo la FR y por ende el trabajo respiratorio.

E1 Tiempo de rampa, se refiere a una característica con que cuentan algunos equipos, en que se da una latencia en minutos para que se logren las presiones seleccionadas por el operador. Generalmente el EPAP mínimo para iniciar el tiempo de rampa es de $4 \mathrm{cmH}_{2} \mathrm{O}$ y el tiempo para alcanzar las presiones planificadas es de 5 a $30 \mathrm{~min}$. Esta modalidad de entrega progresiva de la presión en la vía aérea es particularmente útil y cómoda en pacientes con SAOS, dándole el tiempo suficiente para dormirse ${ }^{4,14,16,22}$.

Opción AVAPS (presión de soporte con volumen promedio asegurado). En esta opción disponible en los Bipap Synchrony (Respironics, USA) permite fijar un VC, mínimo $200 \mathrm{ml}$, en las modalidades S, S/T y PC. El equipo realiza una ventilometria de los volúmenes exhalatorios y ajusta el nivel de IPAP en el rango de 2 valores prefijados (IPAP máximo y mínimo), de esta manera si el VC es inferior durante los ciclos posteriores se aumentara el IPAP necesario para lograr este VC objetivo hasta un valor no superior al IPAP máximo. Si esto sucede y no se logra el VC, se activa la alarma de VC no logrado. Es muy útil para prevenir el riesgo de hipoventilación, manteniendo todas las ventajas de la ventilación ciclada por tiempo y limitada por presión ${ }^{22}$.

\section{Elección de la modalidad ventilatoria}

Existe poca evidencia que sugiera la modalidad a emplear en distintas patologías; en general se utiliza la mejor tolerada por el paciente ${ }^{14,16}$.

En insuficiencia respiratoria aguda se prefiere el BIPAP en sus modos $\mathrm{S}$ y $\mathrm{S} / \mathrm{T}$, logra mejorar la ventilación minuto, disminuye la FR y el trabajo respiratorio; mejora la gasometria y reduce la tasa de intubación ${ }^{3-7,23}$. La excepción es el edema pulmonar agudo cardiogénico, donde se podría utilizar tanto el CPAP y BIPAP en su modo S, la mayoría de los autores prefieren el CPAP, logrando una menor FR, corregir la acidosis respiratoria y mejorar la hemodina$\operatorname{mia}^{10,17,23}$.

En insuficiencia crónica reagudizada, se prefiere el BIPAP en su modo $\mathrm{S} / \mathrm{T}$ o $\mathrm{P} / \mathrm{C}$, con la que se logra mejorar la ventilación minuto, FR y gasometría g, $^{7,23,24}$.

\section{Equipos para entrega de AVNI: Ventiladores mecánicos}

La AVNI utiliza modos ventilatorios limitados por presión, lo que implica fijar límites de presión inspiratoria y espiratoria. Existen generadores de flujo exclusivos para AVNI, más económicos que los respiradores a presión positiva convencionales. Entre ellos destacan los equipos de BiPAP o bi-level, transportables, eléctricos y de flujo continuo, que generan presión a través de un compresor. Estos permiten reconocer y compensar las fugas involuntarias del sistema; los equipos más tradicionales gatillan el flujo inspiratorio para entregar la IPAP programada cuando sensan la generación de flujo por el paciente en el sistema de $40 \mathrm{ml} / \mathrm{s}$. por más de $30 \mathrm{~ms}$., el término de la inspiración se produce cuando esta se prolonga por mas de 3 S. o el flujo ha descendido a $1 / 4$ del flujo máximo. Los equipos Vipap II y III (Resmed, USA) pueden ser programados para la entrega de un tiempo inspiratorio mínimo y máximo y los equipos S/T-D 30, Vision, Harmony y Syncrhony (Respironics, USA) poseen un algoritmo de sensibilidad definido como Auto-Track que detecta el patrón de flujo del paciente ajustando automáticamente los umbrales de sensibilidad a través de tres algoritmos $\left.{ }^{22}: 1\right)$ Umbral de trigger inspiratorio de $6 \mathrm{ml} / \mathrm{s}$. en un periodo de $30 \mathrm{~ms}$.; 2) Algoritmo de disparo determinado por la forma de la curva de flujo de IPAP a EPAP y EPAP a IPAP; 3) Trigger según umbral espiratorio espontáneo para ciclar a EPAP.

La AVNI, puede además realizarse utilizando ventiladores mecánicos convencionales con microprocesador, que cuenten con la modali- 
dad de presión de soporte (New Port Wave o New Port E500, USA), o aquellos con modalidad no invasiva incorporada (Evita de Dräger, Vela de Vyasis, I-Vent de Versamed). La administración de AVNI a través de un ventilador convencional permite determinar la concentración inspirada de oxígeno, prevenir la respiración de aire exhalado por el uso de doble tubuladura y usar los monitores y alarmas del ventilador ${ }^{22,25}$.

La elección del respirador para la administración de VNI dependerá de la variedad y características particulares de los equipos disponibles, tipo de paciente, ámbito en que se aplique y experiencia del operador.

\section{Interfases}

La elección de la interfase apropiada es fundamental para lograr una adecuada transferencia de presiones a la vía aérea, lo que se traduce en una ventilación apropiada y exenta de efectos colaterales indeseados, como lesiones en los puntos de apoyo ${ }^{3,7,15,17}$. Las interfases deben ser de material blando, flexible, siliconado, transparentes, con la superficie de adaptación lisa y acolchada (inflable o material tipo gel).

La mascarilla nasal es la mejor tolerada y suele ser la elección en los pacientes con AVNI en domicilio ${ }^{15,26}$. La mascarilla nasobucal se prefiere en respiradores bucales y en pacientes con insuficiencia respiratoria aguda con parámetros ventilatorios altos. La mascarilla facial completa es infrecuentemente utilizada en pediatría, siendo una alternativa para niños mayores de 2 años con alteraciones en su morfología craneofacial o con lesiones en los puntos de apoyo ${ }^{26}$.

Las interfases existentes deben ser fijadas mediante sistemas elásticos, impidiendo las fugas, pero a la vez, permitiendo que el paciente esté lo más confortable posible sin producir una compresión exagerada ${ }^{26,27}$.

\section{Sistemas de termohumedificación}

La humedificación de los gases inspirados es esencial en el tratamiento de pacientes que necesitan aporte de gases medicinales, oxígenoterapia, ventilación mecánica invasiva (VMI) y AVNI $^{27,28}$.

El sistema del transporte mucociliar es probablemente la función respiratoria más sen- sible a cambios en la humedad y temperatura del gas inspirado. Las secreciones secas pueden conducir a alteraciones de la actividad ciliar, cambios inflamatorios y necrosis del epitelio ciliado pulmonar, retención de secreciones viscosas y adherentes con impactación secundaria, colonización bacteriana, atelectasias y neumonía ${ }^{29}$.

En la insuficiencia respiratoria aguda la humidificación ineficaz o insuficiente estaría relacionada directamente con el fracaso de la $\mathrm{AVNI}^{2,4}$. Por ello en los pacientes con AVNI mayor a $12 \mathrm{~h}$ continuas es necesario establecer una eficiente termohumedificación de la vía aérea con sistemas tradicionales tipo sobrepaso o aquellos ad-hoc compatibles con los generadores de flujo ${ }^{28,29}$.

El uso de humedificación pasiva con intercambiadores de humedad y temperatura (HME) no son recomendables para la entrega de AVNI con generadores de flujo. Aumentan la resistencia en el sistema, altera la respuesta de los trigger y entregan un acondicionamiento del aire inspirado insuficiente ${ }^{30}$.

\section{Oxígenoterapia}

Se aportará el $\mathrm{O}_{2}$ necesario para $\mathrm{SpO}_{2}$ mayor o igual a $93 \%$. Los generadores de flujo no tienen un mezclador interno, por este motivo la entrega de $\mathrm{FiO}_{2}$ será variable dependiendo de los flujos entregados por las presiones programadas y del escape generado. La mejor alternativa para entregar $\mathrm{O}_{2}$ es por medio de una conexión en $\mathrm{T}$ colocada a la salida del Bipap, previo a la conexión a la tubuladura; que puede servir como reservorio y determinar una $\mathrm{FiO}_{2}$ más estable que dependerá de la mezcla producida durante el ciclo inspiratorio y espiratorio entre el flujo de $\mathrm{O}_{2}$ desde su fuente de administración (concentrador, cilindro, red) y el flujo generado por el $\mathrm{BiPAP}^{31}$. Con flujos de $\mathrm{O}_{2}$ menores de $3 \mathrm{l} / \mathrm{min}$ generalmente solo es necesario usar un humedificador tipo burbuja, en caso de flujos mayores podría ser necesario sistemas de termohumedificación tradicionales tipo sobrepaso o aquellos compatibles con los generadores de flujo ${ }^{29,31}$. El escape de flujo a través de la interfase es causa frecuente de desaturación, que no será corregida con aumentos en la concentración de $\mathrm{O}_{2}$, sino reposi- 
cionando y adaptando la mascarilla a la cara del paciente ${ }^{27,31}$.

\section{Terapia broncodilatadora}

En pacientes agudos inestables, puede realizarse nebulización continua con salbutamol 0,3 a $0,5 \mathrm{mg} / \mathrm{kg} / \mathrm{h}(0,05$ a $0,1 \mathrm{ml} \times \mathrm{kg} \times \mathrm{h}$ de salbutamol al $0,5 \%, 1 \mathrm{cc}=5 \mathrm{mg}$ ) solo o adicionando bromuro de ipatropio, $0,25 \mathrm{a} 0,5 \mathrm{mg}$, con un nebulizador tipo Hudson Draft II. La infusión puede ser calculada para $4 \mathrm{~h}$, con un volumen total de $72 \mathrm{ml}$ completado con solución fisiológica al 0,9\%, empleando un microgoteo o bomba de infusión continua que asegure un debito horario de $18 \mathrm{ml} / \mathrm{h}(0,3 \mathrm{ml} / \mathrm{min})^{32}$. Puede instalarse un sistema de nebulización entre la conexión al paciente y el orificio de fuga espiratorio $^{27,32}$.

Es posible también, agregar al circuito sistemas de inhalación con cámara espaciadora o con adaptadores para inhaladores ${ }^{27,32,33}$. Estos sistemas deben disparar la carga del inhalador en sentido contrario al paciente, con objeto de reducir la impactación del fármaco en el circuito y para coordinar el inicio de la inspiración con la administración del fármaco.

Pese a estas alternativas de aerosolterapia en AVNI, no hay trabajos confiables que demuestren la distribución del fármaco con estos sistemas, motivo por lo cual, sigue siendo la forma más confiable el empleo de inhaladores de dosis medida y aerocámara durante los periodos de ventana ${ }^{32,33}$.

\section{Asistencia ventilatoria no invasiva en el paciente crónico}

\section{Utilidad de la AVNI en pacientes crónicos}

A partir de estudios fisiológicos en adultos se ha demostrado que el soporte ventilatorio no invasivo logra disminuir la actividad electromiográfica del diafragma, la presión transdiafragmática, la FR y aumentar el VC, lo que se traduce en un menor trabajo respiratorio y aumento de la ventilación minuto, revertiendo la hipoventilación crónica ${ }^{3}$. Dentro de los principales beneficios de la AVNI en el paciente crónico se encuentra:

- Estabilidad de la vía aérea superior, lo que es especialmente significativo durante el sueño, permitiendo un flujo aéreo normal, con mínima resistencia y por lo tanto, menor trabajo respiratorio, sumado a una mejoría en la arquitectura del sueño $0^{12,23,34}$. Esto justifica su empleo en pacientes con SAOS y aquellos con síndromes malformativos craneofaciales.

- Menor consumo de $\mathrm{O}_{2}$ al disminuir el trabajo respiratorio, menor producción de $\mathrm{CO}_{2} \mathrm{y}$ gasto energético por parte de los mismos músculos respiratorios. A este beneficio se agrega el aumento de la resistencia a la fatiga durante el día cuando se usa el soporte ventilatorio durante la noche $e^{11,34,35}$.

- Aumento de la CRF en pacientes con limitación ventilatoria restrictiva al entregar soporte de la presión transpulmonar, con lo que se obtiene una menor predisposición a la hipoxemia y menores requerimientos de $\mathrm{O}_{2}$ al disminuir la tendencia al colapso alveolar (atelectasias) y mejorar la relación ventilación perfusión. El aumento que produce en la distensibilidad pulmonar optimiza la mecánica del sistema pulmón-tórax, mejorando el intercambio gaseoso $1,11,34,35$.

El uso sostenido de esta terapia en pacientes portadores de dicha condición mejora la sensibilidad del centro respiratorio al $\mathrm{CO}_{2}{ }^{19}$. El paciente con insuficiencia ventilatoria crónica que recibe soporte ventilatorio no invasivo presenta menos exacerbaciones agudas y menos hospitalizaciones ${ }^{35}$. Al disminuir los umbrales de fatigabilidad y descargar la sobrecarga de trabajo respiratorio se logra una condición basal que enfrente mejor las descompensaciones, lo que lleva a una mejoría en la calidad de vida ${ }^{35,36}$. La AVNI en el paciente crónico se entrega utilizando fundamentalmente generadores de flujo y presión positiva en dos niveles (Bipap) en el domicilio en pacientes con patologías restrictivas con hipoventilación nocturna secundaria a insuficiencia de la bomba respiratoria por enfermedades neuromusculares y cifoescoliosis ${ }^{9,11-}$ 13,15,18-20. Se utiliza también en niños con fibrosis quística u otras formas de daño pulmonar crónico, síndrome de hipoventilación central, apneas obstructivas del sueño y obstrucción de la vía aérea superior ${ }^{15,37,38}$ (tabla 1 ). 
Tabla 1. Causas de insuficiencia respiratoria crónica susceptibles de AVNI

1. Enfermedades del sistema nervioso central

a. Malformación de Arnold Chiari

b. Traumatismos y alteraciones vasculares

c. Trastornos del control ventilatorio

d. Mielomenigocele

2. Enfermedades neuromusculares

a. Distrofias musculares

b. Atrofia muscular espinal

c. Miopatías congénitas

3. Anormalidades de la caja torácica

a. Cifoescoliosis

b. Deformidades de la pared torácica

4. Obstrucción crónica de la vía aérea superior

a. Apnea obstructiva del sueño (SAOS)

b. Sindromes craneofaciales

c. Traqueomalacia

5. Enfermedad pulmonar crónica

a. Fibrosis quística

b. Enfermedad pulmonar crónica post viral

c. Displasia broncopulmonar

6. Síndromes de hipoventilación-obesidad

a. Síndrome de Prader Willi

b. Obesidad mórbida con SAOS

\section{Criterios de selección}

El momento adecuado para iniciar la AVNI a presión positiva es un tema actualmente en revisión. En pacientes con enfermedades neuromusculares y cifoescoliosis, la AVNI introducida al momento de pesquisar hipoventilación durante el sueño mejorará la evolución clíni$\mathrm{ca}^{12,18,38}$

Desde el punto de vista clínico, los pacientes con síndromes de hipoventilación nocturna pueden referir síntomas como sudoración nocturna, cefalea matinal, problemas cognitivos con bajo rendimiento escolar, infecciones respiratorias recurrentes o mal incremento pondoestatural $^{12,19,20,37}$. Los criterios clásicos de selección publicados para niños se han adaptado en base a la experiencia adquirida en adultos y de acuerdo a recomendaciones de expertos ${ }^{14,18,37-39}$. Los criterios considerados por el Programa Chileno de Asistencia Ventilatoria No Invasiva Domiciliaria (www.avni.cl) se muestran en la tabla $2^{37}$. Aquellos pacientes seleccionados como candidatos van a requerir estudios complementarios que permitirán definir la inclusión al programa para la entrega de AVNI y/o en- trenamiento muscular si corresponde ${ }^{(37,38)}$. Estos estudios incluyen: 1) Parámetros de función pulmonar: espirometría y curva flujo volumen; 2) evaluación de fuerza muscular: presión inspiratoria máxima (Pimax) y presión espiratoria máxima (Pemax), ventilación voluntaria máxima (VVM), peak flow tos (pico máximo flujo de tos), evaluación de la fatigabilidad o resistencia a través del tiempo límite (Tlim); 3) estudios durante el sueño: Saturometría continua de pulso de 8 a 12 horas, polisomnografía o poligrafia; 4) monitorización de intercambio gaseoso: Gasometría arterial o venosa, $\mathrm{SpO}_{2}$ diurna y nocturna, Capnografía, registro de $\mathrm{PCO}_{2}$ transcutánea $\left(\mathrm{PtCO}_{2}\right)^{30}$ y 5) evaluación de la deformidad esquelética: Estudio radiológico de columna total (Determinación ángulo de Coobs).

La disminución de la fuerza y resistencia muscular frente a la fatiga es el primer elemento clínico presente en las enfermedades neuromusculares. Lo primero en comprometerse desde el punto de vista funcional serán por lo tanto las presiones máximas generadas por la bomba respiratoria (Pimax y Pemax) y la resistencia (Tlim). Posteriormente ocurre una disminución progresiva de la VVM hasta finalmente afectar los volúmenes pulmonares y la capacidad vital forzada (CVF). Una disminución de la fuerza muscular inspiratoria del $50 \%$ se traducirá en una reducción del $20 \%$ de la CVF por lo que este índice es poco sensible para detectar compromiso inicial de la bomba respiratoria ${ }^{11,19,38}$.

\section{Intervenciones complementarias ${ }^{37}$}

El enfoque multidisciplinario debería incluir:

1. Evaluación por neurólogo: define diagnóstico sindromático y localizatorio, como también velocidad de progresión de la enfermedad y pronóstico.

2. Enfermera: Seguimiento y supervisión de la AVNI. Educación para el autocuidado en condiciones de cronicidad con enfoque familiar.

3. Kinesiólogo y terapista ventilatorio: Seguimiento, plan kinésico respiratorio determinando ingreso a programa de entrenamiento muscular respiratorio.

4. Ortopedista: necesidad de órtesis, artrodesis, vías de abordaje.

5. Fisiatra: evaluación para la rehabilitación 
Tabla 2. Criterios de Selección para asistencia ventilatoria no Invasiva en domicilio

\begin{tabular}{|c|c|c|}
\hline Criterios de selección & Inclusión & Exclusión \\
\hline $\begin{array}{l}\text { Condición clínica estable, sin cambios } \\
\text { importantes de los parámetros de AVNI } \\
\text { en las últimas tres semanas }\end{array}$ & $\begin{array}{l}\text { Pacientes con insuficiencia respiratoria } \\
\text { crónica y síndromes de hipoventilación } \\
\text { nocturna secundarios a patología que } \\
\text { cumplan los siguientes criterios: } \\
\text { Saturometría nocturna contínua } \\
\text { anormal }\left(\mathrm{SpO}_{2}<90 \%>10 \% \text { del }\right. \\
\text { tiempo de sueño) } \\
\mathrm{CVF}<50 \% \text { valor predicho, Pimax }<40 \\
\mathrm{CmH}_{2} \mathrm{O}, \mathrm{Peak}_{\text {flow }} \text { tos bajo } 150 \mathrm{l} / \mathrm{m} \\
\mathrm{GSA}: \mathrm{PaCO}_{2}>50 \mathrm{mmHg}, \mathrm{EB}>4 \mathrm{mEq} / \mathrm{l}\end{array}$ & $\begin{array}{l}\text { Enfermedad neuromuscular de progresión } \\
\text { rápida }\end{array}$ \\
\hline $\begin{array}{l}\text { Necesidad de soporte ventilatorio } \\
\text { nocturno por tiempos de permanencia } \\
\text { no mayor a } 10 \text { horas }\end{array}$ & $\begin{array}{l}\text { Pacientes con enfermedad neuromus- } \\
\text { cular de progresión lenta o estacionaria }\end{array}$ & $\begin{array}{l}\text { Trastorno de deglución con ausencia de } \\
\text { protección glótica }\end{array}$ \\
\hline Hipercapnia sin acidosis respiratoria & $\begin{array}{l}\text { Pacientes con compromiso primario o } \\
\text { secundario del comando ventilatorio } \\
\text { y SAOS }\end{array}$ & $\begin{array}{l}\text { Necesidad de soporte ventilatorio por } \\
\text { más de } 10 \text { horas o ausencia de } \\
\text { autonomía respiratoria fuera de soporte } \\
\text { de presión positiva }\end{array}$ \\
\hline IPAP/EPAP no superior a $20 / 8 \mathrm{~cm} \mathrm{H}_{2} \mathrm{O}$ & & Menor de 6 meses (relativo) \\
\hline
\end{tabular}

neurológica. Definición de ayudas técnicas, ortesis, sitting para el manejo de la cifoescioliosis.

6. Fonoaudiólogo: Evaluación y rehabilitación de los trastornos de deglución.

7. Equipo de salud mental: reinserción social, escolar, calidad de vida.

8. Asistente social: informe social, red de apoyo y coordinación intersectorial.

\section{Control ambulatorio ${ }^{27,38}$}

1. Control mensual, trimestral, semestral de acuerdo a evolución. Clínico y funcional.

2. Verificar adherencia y tolerancia al sistema.

3. Registrar acontecimientos adversos.

4. Detectar complicaciones: Edema puente nasal, lesión por compresión (5-10\%), intolerancia a mascarilla o interfase (20-25\%), distensión gástrica y/o irritación ocular.

\section{Estudios de seguimiento}

Existen pocos estudios de seguimiento de pacientes crónicos con AVNI domiciliaria $^{18,20,34,39,40}$. El inicio de la VNI nocturna en pacientes con síndrome de hipoventilación nocturna se asocia a una mayor eficacia del sueño, menor proporción de sueño superficial en favor del aumento del sueño más profundo, y la ten- dencia a un sueño REM más prolongado con una disminución significativa en los despertares nocturnos ${ }^{17,25,40}$. La presencia de síntomas diurnos como cefaleas matinales, falta de apetito y alteración en la concentración mejoraron en la mayoría de los pacientes ${ }^{20,34,39,40}$. Deben evaluarse periódicamente parámetros de función pulmonar y de intercambio gaseoso.

\section{Asistencia ventilatoria no invasiva en el paciente con insuficiencia respiratoria aguda}

\section{Utilidad de la AVNI en pacientes agudos.}

En estos casos, los objetivos de la AVNI son: disminuir el uso de VMI y evitar sus complicaciones, facilitar la extubación, reducir días de hospitalización, morbilidad asociada y optimizar los recursos en la red asistencial ${ }^{3,5,7,35,41}$. Posee una serie de ventajas sobre la VMI: mejor tolerada, fácil de instalar y retirar, preserva la tos, fonación y alimentación; puede implementarse tanto en unidades de cuidados intensivos, como cuidados intermedios, con personal y monitorización adecuados ${ }^{3,5-7}$.

Se ha usado con éxito en niños con patologías agudas, en estudios retrospectivos o series clínicas pequeñas ${ }^{1,2,4,7,8,35}$. No existen estudios 
que comparen los beneficios de la AVNI con la ventilación mecánica convencional, sin embargo en menores con insuficiencia respiratoria aguda se ha usado previo al fallo ventilatorio global, con vía aérea y hemodinamía estables y sin compromiso sensorial ${ }^{7}$; reportándose disminución de los días de hospitalización, sin demostrar claramente que disminuya el riesgo de intubación ${ }^{2,7,35,41}$. Del mismo modo la AVNI ha sido útil al término de la ventilación mecánica invasiva, disminuyendo la falla de extubación ${ }^{41}$. En el caso de asma grave los estudios muestran resultados dispares, posiblemente dado lo heterogéneo en la selección de pacientes ${ }^{24}$.

La tabla 3 presenta las indicaciones de AVNI en patologías agudas, incluyendo sus dos grupos diana: insuficiencia respiratoria aguda e insuficiencia respiratoria crónica reagudiza$\mathrm{da}^{3,6,7,35,36,41}$.

Para seleccionar al paciente, es necesario definir criterios de inclusión clínicos y gasométricos. La presencia de uno o más de los siguientes puntos determinan la posibilidad de empleo de AVNI ${ }^{1,3,6}$ :

1. Disnea moderada a severa con aumento del trabajo respiratorio (uso de musculatura accesoria) y FR mayor al límite superior para la edad.

2. Necesidad de $\mathrm{FiO}_{2}>40 \%$ y saturación $\mathrm{Hb}$ $<$ de $93 \%$ o $\mathrm{PaFi}<150$

3. Hipercapnea con $\mathrm{PCO}_{2}>45 \mathrm{mmHg}$ y $\mathrm{pH}$ no inferior de 7,25.

4. Progresión del compromiso pulmonar clínico y radiológico.

La VNI debe ser indicada con precaución, debe existir estricta vigilancia. En pacientes inmunosuprimidos, la VNI asociada al tratamiento convencional puede reducir la mortali$\operatorname{dad}^{3,5,21,41}$. Se sugiere la indicación precoz en los pacientes con enfermedad neuromuscular y/o cifoescoliosis ${ }^{23,27,35}$.

Los criterios de exclusión o contraindicaciones son: indicación de intubación, vía aérea inestable, falla orgánica múltiple, inestabilidad hemodinámica, compromiso de conciencia (Glasgow $<10$ ), neumotórax o neumomediastino, ausencia de reflejo de tos o nauseoso, vómitos, cirugía, trauma o deformidad facial, alto riesgo de aspiración e intolerancia al método ${ }^{4,5,7}$. In-

Tabla 3. Indicaciones de AVNI en patologías agudas

\begin{tabular}{ll}
\hline Insuficiencia respiratoria aguda & Insuficiencia respiratoria crónica reagudizada \\
\hline Infección respiratoria aguda baja & Enfermedades neuromusculares: atrofia espinal, Duchenne \\
Crisis asmática/bronquiolitis & Cifoescoliosis \\
Atelectasias & Fibrosis quística \\
IRA en paciente inmunodeprimido & Daño pulmonar crónico \\
Edema pulmonar cardiogénico y no cardiogénico & \\
Post extubación (weaning ) & \\
Apoyo post anestesia & \\
\hline
\end{tabular}

Tabla 4. Predictores de éxito y fracaso en insuficiencia respiratoria aguda

\begin{tabular}{ll}
\hline Criterios de éxito & Criterios de fracaso \\
\hline - Menor severidad de enfermedad (PRIMS) & - Compromiso de conciencia \\
- Paciente cooperador, ausencia compromiso sensorial. & - No aceptación del método por el paciente y/o los padres \\
Apropiada sincronía paciente-ventilador & - Imposibilidad de acoplar o falta de tolerancia al sistema de \\
- Menor fuga de aire & AVNI \\
- Hipercapnia sin acidosis respiratoria severa pH $>7,25$ & - Deterioro rápidamente progresivo con necesidad de \\
- Mejoría oxigenación, frecuencia cardiaca y respiratoria & protección de vía aérea \\
en primeras 2 horas de VNI & Hipercapnía con acidosis respiratoria (pH $<7,20)$ \\
& - Incapacidad de mejorar los síntomas, parámetros de \\
& oxigenación yentilación previos al inicio de la AVNI, \\
& dentro de las dos horas de su inicio
\end{tabular}


vestigaciones sobre este tema han determinado predictores de éxito en insuficiencia respiratoria aguda (tabla 4$)^{3-5,7}$.

\section{Monitorización de la AVNI en el paciente agudo}

Monitorización clínica ${ }^{\mathbf{5 , 6 , 8 , 2 7}}$

Las primeras $4 \mathrm{~h}$ son fundamentales para establecer la adaptación, evaluable generalmente dentro de los 30 min de inicio, y el éxito de la VNI. Es necesario evaluar:

1. Adaptación al equipo, interfase (mascarilla) y arnés.

2. Distensión abdominal. Algunos pacientes requieren instalación de SNG a caída libre.

3. FR, trabajo respiratorio y la actividad de los músculos accesorios (incluida la presencia de respiración paradojal), éstos deben disminuir en el plazo de 1 a $2 \mathrm{~h}$.

4. Movilidad torácica y ventilación en función de la apropiada auscultación del murmullo pulmonar en los planos axilares y posteriores. Permite ajustar el nivel de IPAP necesario. Permite seguir la evolución de la patología que condicionó la indicación de VNI.

5. Estado de conciencia: aletargamiento o agitación.

6. FC y Presión arterial.

7. Rx. Tórax, previo al inicio de la AVNI, y luego de acuerdo a la evolución del paciente. Sirve para evaluar la patología de base, nivel de EPAP necesario, los efectos de la AVNI, la presencia de atelectasias y edema pulmonar.

8. Monitorización de efectos adversos

\section{Monitorización gasométrica $a^{5,6,8,27}$}

Los GSA o GSV están indicados cuando existe sospecha de hipoventilación, en insuficiencia respiratoria global o en aquellos pacientes en que no se logra mantener la $\mathrm{SpO}_{2}>$ de $90 \%$.Cambios significativos en los gases sanguíneos se registran dentro de las primeras 2-4 $\mathrm{h}$ del inicio de la terapia. Salvo signos de empeoramiento, es recomendable posponer el control de ellos no antes de la primera hora.

\section{Monitorización del equipo}

Es importante mantener un chequeo permanentemente de los parámetros ventilatorios, para establecer cambios según la evolución del paciente $^{5,6,27}$. Algunos equipos permiten registrar la FR total lograda, el VC y $\mathrm{Q}_{\min }$ exhalatorio, el flujo de escape $\left(\mathrm{Q}_{\text {leak }}\right)$, IPAP, EPAP, Ti o \% de Ti, Rise time, Tiempo de rampa. El VC idealmente debe permanecer entre 7 a $10 \mathrm{cc} / \mathrm{kg}$ y el $\mathrm{Q}_{\text {leak }}$ no debe ser mayor al 30\% del flujo total del sistema ${ }^{17,22,25,37}$.

\section{Inconvenientes y complicaciones de la AVNI ${ }^{5,6,27}$}

En pediatría, el principal inconveniente del uso de esta técnica es que requiere mayor atención para ajustar la mascarilla, minimizar las fugas y de este modo, conseguir la mejor adaptación y tolerancia del paciente p $^{3,6,25,27}$.

Las complicaciones asociadas a la AVNI son en general, menos importantes que las producidas por la VMI $\mathrm{VM}^{3-7,23,27,38}$. Las más frecuentes están en relación con la interfase y su ajuste. Su prevención y corrección pasan por la adecuada selección de las mascarillas y un apropiado cuidado de enfermería, en especial de los sitios de apoyo ${ }^{26,27}$.

a) Hipoventilación e Hipercapnia: Se deben usar interfases con el menor espacio muerto posible y no ocluir los portales de exhalación. Es importante tener una gradiente de presión apropiada para la transferencia esperada de VC.

b) Intolerancia a la mascarilla: Situación que se presenta con mayor frecuencia en niños pequeños. Puede deberse a una mala adaptación por una desproporción entre la mascarilla y la forma del macizo craneofacial, por escape del flujo, por una mala fijación o por escape a través de la boca en pacientes con mascarillas nasales, que obligue a los equipos a aumentar los flujos para mantener la presión seleccionada.

c) Conjuntivitis por escape de flujo aéreo o compresión por los bordes de apoyo.

d) Lesiones de la piel en los puntos de apoyo, desde irritación hasta escaras y cicatrices.

e) Distensión gástrica y aspiración alimentaria.

f) Alteraciones del crecimiento del macizo facial, como la hipoplasia mediofacial. Puede presentarse en niños ventilados en forma crónica y prolongada. 
g) Neumotórax, neumomediastino: Infrecuente, podría aparecer en pacientes con requerimientos altos de presión.

h) Otras son inherentes al tipo de soporte ventilatorio y se logran prevenir utilizando los parámetros mínimos que logren satisfacer los objetivos clínicos, dentro de estos se encuentran: sequedad de mucosas, rinitis vasomotora y despertares nocturnos producidos por el flujo de aíre del equipo.

\section{Factores vinculados al fracaso de la $A V N I$}

Las condiciones vinculadas al fracaso de la AVNI pueden ser exploradas a través de las siguientes preguntas: ¿hay fugas en la interfase?, ¿hay atelectasias por hipoventilación en las zonas dependientes?, ¿es necesario intensificar la kinesiterapia respiratoria?, ¿la elección del paciente fue la apropiada?, ¿es un enfermo candidato a ventilación convencional?, ¿hay complicaciones como distensión gástrica, erosiones o lesiones en el puente nasal, existe aumento de la resistencia en la vía aérea por broncoespasmo o menos frecuentemente se ha producido un neumotórax?, ¿Es necesario aumentar el EPAP o modificar la modalidad ventilatoria, el tiempo inspiratoria o el Rise-time?.

Una ponderación de los aspectos fisiopatológicos y clínicos realizada caso a caso permitirán lograr un soporte ventilatorio exitoso, evaluable en base a los objetivos generales como es mejorar la calidad de vida y prolongar ésta cuando sea posible y aquellos específicos como es asistir la musculatura respiratoria, disminuir la hipercapnia nocturna, mejorar el intercambio gaseoso, prevenir las atelectasias y mejorar la permeabilidad de la vía aérea superior $^{17,23,38}$. La tabla 4 resume los criterios de fracaso de AVNI en paciente agudo ${ }^{6,7,27}$.

\section{Retirada de AVNI en paciente agudo ${ }^{5,6,41}$}

No está claramente definido el mejor método de retirada de AVNI. Es aconsejable bajar primero el aporte del flujo de oxigeno, idealmente a menos de $31 / \mathrm{m}$, luego disminuir según tolerancia la presión de soporte, minimizando el diferencial entre la IPAP y EPAP hasta dejar en CPAP con presiones de 5 a $6 \mathrm{cmH}_{2} \mathrm{O}$. También es posible realizar ventanas sin AVNI, progresivas, hasta el retiro completo de la AVNI ${ }^{6,735}$. Es aconsejable mantener el apoyo ventilatorio nocturno como último eslabón antes de la suspensión de la AVNI ${ }^{3,6}$. En aquellos pacientes con enfermedades neuromusculares, cifoescoliosis o insuficiencia respiratoria crónica en etapas avanzadas es importante considerar la necesidad de incluir en AVNI en domici$\operatorname{lio}^{3,6,23,35}$.

Los parámetros para determinar retiro de la VMNI son:

1. Mejoría de la condición clínica, resolución de las apneas, disminución de la disnea, aumento de la fuerza muscular y mejoría de la oxigenación con $\mathrm{SpO}_{2}$ estable > de 93\% con $\mathrm{FiO}_{2}<40 \%$ (evaluado sin AVNI).

2. Mejoría del trabajo respiratorio evaluado por la disminución de la FR.

3. Mejoría de los índices de ventilación con eucapnea en pacientes sin insuficiencia respiratoria crónica hipercápnica y $\mathrm{PaCO}_{2}$ en rango de su nivel histórico, para aquellos pacientes hipercapnicos.

4. Mejoría radiológica con resolución de las atelectasias, disminución o desaparición de las imágenes parenquimatosas pulmonares y presencia de a lo menos 7 espacios intercostales en cada campo pulmonar.

5. Regreso a parámetros habituales de ventilación y de condición medica en los pacientes con AVNI domiciliaria.

\section{Conclusiones}

La AVNI es una alternativa a la VMI en pacientes que evolucionen con insuficiencia respiratoria aguda y crónica, que cumplen con criterios de selección. Para ello, se requiere una monitorización básica implementable en unidades de cuidado intensivo e intermedio; así como también en el domicilio, escenario del paciente crónico, que incluye monitorización de parámetros clínicos, supervisión de profesional y educación continua al paciente y familia. En el grupo de niños con enfermedades respiratorias crónicas, es útil especialmente en enfermedades neuromusculares, cifoescoliosis, SAOS y fibrosis quística; pudiendo alterar la historia natural de la enfermedad al iniciarse al momen- 
to de identificar la hipoventilación nocturna, sin esperar al deterioro clínico funcional ya evidente en vigilia.

\section{Agradecimientos}

A los pacientes y familias del Programa Chileno de Asistencia Ventilatoria No Invasiva en Domicilio y del Hospital Josefina Martínez. A los autores de las Guías de Ventilación Mecánica Prolongada: María Lina Boza, Guisela Moya, Guisela Villarroel, Rebeca Paiva y Andrés Koppmann.

\section{Referencias}

1.- Fortenberry JD, Del Toro J, Jefferson LS, Evey L, Haase D: Management of Pediatric Acute Hipoxemic Respiratory Insufficiency With Bilevel Positive Pressure (BIPAP) Nasal Mask Ventilation. Chest 1995; 108: 1059-64.

2.- Birnkrant DJ: Consensus Conference Report; COPD, and Nocturnal Hypoventilation. A Failure Due to Restrictive Lung Disease; Pressure Ventilation in Chronic Respiratory; Clinical Indications for Noninvasive Positive. Chest 1999; 116:521-534.

3.- Prado F, Godoy MA, Godoy M: Ventilación no invasiva como tratamiento de la insuficiencia respiratoria aguda en Pediatría. Rev Méd Chile 2005; 133: 525-33.

4.- Akingbola OA, Hopkins RL: Pediatric noninvasive positive pressure ventilation. Pediatr Crit Care Med 2001; 2: 164-9.

5.- Caples SM, Gay PC: Noninvasive positive pressure ventilation in the intensive care unit: A concise review. Crit Care Med 2005; 33: 2651-8.

6.- Medina A, Prieto S, Rey M, Concha A, Menéndez S, Crespo M: Aplicación de la Ventilación no Invasiva en una Unidad de Cuidados Intensivos Pediatricos. An Pediatr (Barc) 2005; 62: 13-9.

7.- Essouri S, Chevret L, Durand P, Haas V, Fauroux B, Devictor $D$ : Noninvasive positive pressure ventilation: Five years of experience in a pediatric intensive care unit. Pediatr Crit Care Med 2006; 7: 329-34.

8.- Yañez LJ, Yunge M, Emilfork M, et al: A prospective, randomized, controlled trial of noninvasive ventilation in pediatric acute respiratory failure. Pediatr Crit Care Med 2008; 9: 484-8.

9.- Wallgren-Petterssona C, Bushbyb K, Melliesc $U$, Simonds A: Ventilatory Support in Congenital Neuromuscular Disorders-Congenital Myopathies, Congenital Muscular Dystrophies, Congenital Myotonic Dystrophy and SMA (II). Neuromuscular Disorders 2004;14: 56-69.
10.- Norregaard $O$ : Noninvasive ventilation in children. Eur Respir J 2002; 20: 1332-42.

11.- Simonds $A K$ : Recent advances in respiratory care for neuromuscular disease. Chest 2006; 130: 1879-86.

12.- Toussaint M, Chatwin M, Soudon P: Mechanical ventilation in Duchenne patients with chronic respiratory insufficiency: clinical implications of 20 years published experience. Chron Respir Dis 2007; 4: 167-77.

13.- Ward S, Chatwin M, Heather S, Simonds AK: Randomised controlled trial of non-invasive ventilation (NIV) for nocturnal hypoventilation in neuromuscular and chest wall disease patients with daytime normocapnia. Thorax 2005; 60: 1019-24.

14.- Mehta S, Hill NS: Noninvasive Ventilation. Am J Respir Crit Care Med 2001; 163: 540-77.

15.- Sánchez I, Valenzuela A, Bertrand P: Apoyo ventilatorio domiciliario en niños con insuficiencia respiratoria crónica. Experiencia clínica. Rev Chil Pediatr 2002; 73: 51-5.

16.- Kakkar RK, Berry RB: Positive airway pressure treatment for obstructive sleep apnea. Chest 2007; 132: $1057-72$.

17.- Timothy $B$, Op't H: Physiology of Ventilatory Support. In: Egan P.D., Ed., Egan's Fundamentals of Respiratory Care. 2003; 963-1002.

18.- Fauroux B, Lofaso F: Domiciliary non-invasive ventilation in children. Rev Mal Respir 2005; 22: 289 303.

19.- Simonds $A K$ : Respiratory support for the severely handicapped child with neuromuscular disease: ethics and practicality. Semin Respir Crit Care Med 2007; 28: $342-54$

20.- Young HK, Lowe A, Fitzgerald DA, et al: Outcome of noninvasive ventilation in children with neuromuscular disease. Neurology 2007; 68: 198-201.

21.- Teague $W$ : Non-invasive positive pressure ventilation: Current status in paediatric patients. Paediatr Respir Rev 2005; 6: 52-60.

22.- Battisti A, Tassaux D, Janssens JP, Michotte JB, Jaber $S$, Jolliet P: Performance characteristics of 10 home mechanical ventilators in pressure-support mode: a comparative bench study. Chest 2005; 127: 1784-92.

23.- Fauroux B, Lofaso F: Non-invasive mechanical ventilation: when to start for what benefit? Thorax 2005; 60: 979-80.

24.- Soroksky A, Stav D, Isaac Shpirer I: MDA Pilot Prospective, Randomized, Placebo-Controlled Trial of Bilevel Positive Airway Pressure in Acute Asthmatic Attack. Chest 2003; 123: 1018-25.

25.- Prinianakis G, Kondili E, Georgopoulos D. Effects of the flow waveform method of triggering and cycling on patient-ventilator interaction during pressure support. Intensive Care Med 2003; 29: 1950-9.

26.- Hill NS: Saving face: better interfaces for noninvasive ventilation. Intensive Care Med 2002; 28: 227-9.

27.- Hess DR: The evidence for noninvasive positivepressure ventilation in the care of patients in acute respiratory failure: a systematic review of the literature. Respir Care. 2004; 49:810-29. 
28.- Solomita M, Smaldone GC: Humidification and noninvasive ventilation. Respir Care 2007; 52: 24-5.

29.- Lellouche F, Maggiore SM, Deye N, Taillé S, Pigeot $J$, Harf A, Brochard L: Effect of the humidification device on the work of breathing during noninvasive ventilation. Intensive Care Med 2002; 28: 1582-89.

30.- Norreegaard $O$ : Non-invasive ventilation in acute respiratory failure in children. En: Non-invasive respiratory Support. Simonds AK. Third edition, Edward Arnold (Publishers) Ltd. 2007; 257-73.

31.- Thys F, Liistro G, Dozin O, Marion E, Rodenstein D: Determinants of $\mathrm{FiO} 2$ with oxygen supplementation during noninvasive two-level positive pressure ventilation. Eur Respir J 2002; 19: 653-7.

32.- Dhand $R$. Inhalation therapy in invasive and noninvasive mechanical ventilation. Curr Opin Crit Care 2007; 13: 27-38.

33.- Dhand R: Inhalation therapy with metered-dose inhalers and dry powder inhalers in mechanically ventilated patients. Respir Care 2005; 50:1331-34.

34.- Mellies U, Ragette R, Dohna Schwake C, Boehm H: Long-term noninvasive ventilation in children and adolescents with neuromuscular disorders. Eur Respir J 2003; 22: 631-6.
35.- Brochard L, Mancebo J, Elliott $M W$ : Noninvasive ventilation for acute respiratory failure. Eur Respir J 2002; 19: 712-21.

36.- Dohna-Schwake C, Podlewski P, Voit T, Mellies U: Non-invasive ventilation reduces respiratory tract infections in children with neuromuscular disorders. Pediatr Pulmonol 2008; 43: 67-71.

37.- Ministerio de Salud de Chile: Programa Nacional de Asistencia Ventilatoria No Invasiva en Atención Primaria de Salud: Normas Técnicas. Revista Neumología Pediátrica 2007; 2: 38-48.

38.- Prado F., Bertrand P., Vega LE., Sánchez I., Romero $J E$. Asistencia Ventilatoria en Niños. Revista Iberoamericana 2007; 9: 35-42.

39.- Graham R, Fleegler E, Robinson $W$ : Chronic Ventilator Need in the Community: A 2005 Pediatric Census of Massachusetts. Pediatrics 2007; 119; e12807.

40.- Hammer J: Home mechanical; Ventilation in children: indications and practical aspects. Schweiz Med Wochenschr 2000; 130: 1894-902.

41.- Wysocki M, Antonelli M: Noninvasive mechanical ventilation in acute hypoxaemic respiratory failure. Eur Respir J 2001; 18: 209-20. 